\title{
Gesetz über den Europäischen Haftbefehl: verfassungswidrig!
}

EuHbG, Art. 16 GG

BVerfG, 2 BvR 2236/04 v. 18.7.2005 = NJW 2005, 2289 - 2303 m.abw. Meinung Broß, Lübe-Wolff und Gerhardt

Bernd-Rüdeger Sonnen

\section{Sachverhalt (gekürzt)}

egen den Beschwerdeführer (B) besteht ein

JEuropäischer Haftbefehl«, in Madrid erlassen mit dem Vorwurf der Beteiligung an einer kriminellen Vereinigung und Terrorismus. B soll eine Schlüsselfigur im europäischen Teil der AlQaida sein. Das OLG Hamburg erklärte die Auslieferung von B, der die deutsche und die syrische Staatsangehörigkeit besitzt, an Spanien für zulässig und die Justizbehörde der Freien und Hansestadt Hamburg bewilligte diese unter der Bedingung, dass dem B nach rechtskräftiger Verurteilung angeboten werde, ihn für die Vollstreckung nach Deutschland zurück zu überstellen.

Gegen den Beschluss des OLG Hamburg und die Bewilligungsentscheidung richtet sich die erfolgreiche Verfassungsbeschwerde von B.

\section{Urteil}

Das Gesetz zur Umsetzung des Rahmenbeschlusses über den Europäischen Haftbefehl und die Übergabeverfahren zwischen den Mitgliedsstaaten der Europäischen Union (Europäisches Haftbefehlsgesetz (EuHBbG) v. 21. Juli 2004 verstößt gegen Art. 2 I i.V.m. Art. 20 III, Art. 16 II und Art 19 IV des Grundgesetzes und ist nichtig.

Der Beschluss des Hanseatischen Oberlandesgerichts Hamburg vom 23. November 2004 verletzt den Beschwerdeführer in seinem Grundrecht aus Art. 16 II des Grundgesetzes. Der Beschluss wird aufgehoben. Die Sache wird an das Hanseatische Oberlandesgericht zurück verwiesen.

Die Bewilligungsentscheidung der Justizbehörde der Freien und Hansestadt Hamburg v.
24. November 2004 verletzt den Beschwerdeführer in seinen Grundrechten aus Art. 16 II und Art. 19 IV des Grundgesetzes. Die Bewilligungsentscheidung wird aufgehoben.

\section{Leitsätze}

1. Art. 16 GG gewährleistet als Grundrecht mit seinem Ausbürgerungs- und Auslieferungsverbot die besondere Verbindung der Bürger zu der von ihnen getragenen freiheitlichen Rechtsordnung. Der Beziehung des Bürgers zu einem freiheitlichen demokratischen Gemeinwesen entspricht es, dass der Bürger von dieser Vereinigung grundsätzlich nicht ausgeschlossen werden kann.

2. Die in der "Dritten Säule« der Europäischen Union praktizierte Zusammenarbeit einer begrenzten gegenseitigen Anerkennung ist ein auch unter Subsidiaritätsgesichtspunkten (Art. 23 Abs. 1 GG) schonender Weg, um die nationale Identität und Staatlichkeit in einem einheitlichen europäischen Rechtsraum zu wahren.

3. Der Gesetzgeber war beim Erlass des Umsetzungsgesetzes zum Rahmenbeschluss über den Europäischen Haftbefehl verpflichtet, das Ziel des Rahmenbeschlusses so umzusetzen, dass die Einschränkung des Grundrechts auf Auslieferungsfreiheit verhältnismäßig ist. Insbesondere hat der Gesetzgeber über die Beachtung der Wesensgehaltsgarantie hinaus dafür Sorge zu tragen, dass der Eingriff in den Schutzbereich des Art. 16 II GG schonend erfolgt. Dabei muss er beachten, dass mit dem Auslieferungsverbot gerade auch die Grundsätze der Rechtssicherheit und des Vertrauensschutzes für den von einer Auslieferung betroffenen Deutschen gewahrt werden sollen.
4. Das Vertrauen des Verfolgten in die eigene Rechtsordnung ist von Art. 16 II GG in Verbindung mit dem Rechtsstaatsprinzip dann in besonderer Weise geschützt, wenn die dem Auslieferungsersuchen zu Grunde liegende Handlung einen maßgeblichen Inlandsbezug hat.

\section{Erläuterung und Kommentierung}

Nach Art. 16 II GG darf kein Deutscher an das Ausland ausgeliefert werden. Allerdings kann durch Gesetz eine abweichende Regelung für Auslieferungen an einen Mitgliedsstaat der Europäischen Union oder an einen internationalen Gerichtshof getroffen werden, soweit rechtsstaatliche Grundsätze gewahrt sind. Eine solche abweichende und einschränkende Regelung ist durch das Europäische Haftbefehlsgesetz erfolgt, von dem das BVerfG nun sagt:

- Das Gesetz verstößt gegen Grundrechte und ist materiell verfassungswidrig.

- Das Gesetz ist nichtig.

- Die angegriffenen Entscheidungen beruhen auf einer verfassungswidrigen Rechtsgrundlage und sind aufzuheben.

Die drei abweichenden Meinungen kommen $\mathrm{zu}$ unterschiedlichen Ergebnissen. Mit der Mehrheit des Senats (5 Stimmen) teilt auch der Richter Broß die Entscheidung, dass das Europäische Haftbefehlsgesetz nichtig ist. Er sieht aber Mängel in der Begründung hinsichtlich von Straftaten mit maßgeblichem Auslandsbezug. Die Richterin Lübbe-Wolff sieht zwar ebenfalls verfassungsrechtliche Mängel, die ihrer Meinung nach es allerdings nicht rechtfertigen, das Gesetz insgesamt für nichtig zu erklären. In seinem Sondervotum legt der Richter Gerhardt dar, warum er das Urteil in keinem Punkt mittragen könne. Er hätte die Verfassungsbeschwerde von $\mathrm{B}$ zurück gewiesen. 
Im Urteil wird zunächst das (eingeschränkte) Verbot der Auslieferung eigener Staatsangehöriger mit der »Beziehung des Bürgers zu einem freiheitlichen demokratischen Gemeinwesen begründet (vgl. Leitsatz 1). Sodann wird auf die historischen Wurzeln und völkerrechtliche Aspekte hingewiesen. Das Grundrecht des Art. 16 GG mit seinem Ausbürgerungs- und Auslieferungsverbot gründe sich auch auf Erfahrungen aus der neueren deutschen Geschichte zur Zeit der nationalsozialistischen Diktatur und entspreche auch der »seit der französischen Revolution gemeineuropäischen Überzeugung, dass Bürger nur in einer statusrechtlich gesicherten Weise ihre politische und zivilrechtliche Rechtsstellung genießen können «. In ihrem Sondervotum warnt dagegen die Richterin Lübbe-Wolff vor einer solchen Überhöhung des Verbots der Auslieferung: »Der Versuch, es in höheren (quasi naturrechtlichen), tieferen (historischen) und weiteren (völkerrechtlichen) Sphären zu verankern, führt in die Irre.«

Einen hohen Rang hat der grundrechtliche Schutz Deutscher vor Auslieferung »aufgrund seiner besonderen Ausgestaltung in Art. 16 II GG und weil das geschützte Interesse seiner lebensweltlichen Bedeutung nach schwer wiegt«. Gleichzeitig warnt sie vor Missverständnissen hinsichtlich der verfassungsrechtlichen Rechtsstellung Nichtdeutscher und verweist auf Art. 2 I und Art. 2 II 2 GG. Sie seien gegenüber Auslieferung und der damit verbundenen Inhaftierung ebenso schutzwürdig, wenn sie seit langem in Deutschland leben oder sogar hier geboren und aufgewachsen sind (vgl. § 80 III IRG).

Da das Grundrecht auf Auslieferungsfreiheit nicht uneingeschränkt gilt, sind die Einschrän- kungen am Grundsatz der Verhältnismäßigkeit und weiteren rechtsstaatlichen Grundsätzen (wie Rechtssicherheit und Vertrauensschutz) zu messen (Leitsatz 3). Insoweit unterscheidet das BVerfG zwischen Delikten mit maßgeblichem Inlandsbezug (vgl. Leitsatz 4), sog. Distanzdelikten und Delikten mit maßgeblichem Auslandsbezug. Wörtlich heißt es in diesem Zusammenhang: "Das Vertrauen des Verfolgten in die eigene Rechtsordnung ist von Art. 16 II GG in Verbindung mit dem Rechtsstaatsprinzip dann in besonderer Weise geschützt, wenn die dem Auslieferungsersuchen zugrunde liegende Handlung ganz oder teilweise auf deutschem Staatsgebiet, auf deutschen Schiffen und Luftfahrzeugen oder an Orten unter deutscher Hoheitsgewalt begangen wurde. Straftatvorwürfe mit einem insofern maßgeblichen Inlandsbezug sind bei Tatverdächtigen deutschen Staatsangehörigen prinzipiell im Inland durch deutsche Strafermittlungsbehörden aufzuklären. Ein maßgeblicher Inlandsbezug liegt jedenfalls dann vor, wenn wesentliche Teile des Handlungs- und Erfolgsortes auf deutschem Staatsgebiet liegen ...

Anders fällt die Beurteilung aus, wenn die vorgeworfene Tat einen maßgeblichen Auslandsbezug hat ... Der Auslandsbezug ist auch und gerade dann anzunehmen, wenn die Tat von vorn herein eine typische grenzüberschreitende Dimension hat und eine entsprechende Schwere aufweist, wie beim internationalen Terrorismus oder beim organisierten Drogen- und Menschenhandel; wer sich in solche verbrecherische Strukturen einbindet, kann sich auf den Schutz der Staatsangehörigkeit vor Auslieferung nicht in vollem Umfang berufen.
Während in den genannten Fallgestaltungen das Ergebnis der Verhältnismäßigkeitsprüfung in aller Regel vorgezeichnet ist, bedarf es der konkreten Abwägung im Einzelfall, wenn ganz oder teilweise in Deutschland gehandelt worden, der Erfolg aber im Ausland eingetreten ist «.

Während die Ausführungen zur Stufe der Delikte mit überwiegendem Inlandsbezug und der Stufe zu den Distanzdelikten Zustimmung verdienen, ergeben sich Probleme bei der Stufe der Delikte mit überwiegendem Auslandsbezug. So kritisiert der Richter Broß in seinem Sondervotum, dass in diesem Fall ein deutscher Staatsangehöriger ohne jede materielle Einschränkung ausgeliefert werden dürfe, der Verfolgte also insoweit wie ein Schuldiger behandelt werde. Darin sieht er eine Verletzung der im Rechtsstaatsprinzip wurzelnden Unschuldvermutung. Es geht aber wohl gar nicht um die Unschuldvermutung, wenn bei Einbindung in »verbrecherische Strukturen « auf einen geringeren »Schutz der Staatsangehörigkeit vor Auslieferung « geschlossen wird. Die Formulierung klingt vielmehr sehr nach einer Sonderregelung unter dem Aspekt (eines in jeder Hinsicht abzulehnenden) Feindstrafrechts.

Aufgrund der Verfassungswidrigkeit ist also ein neues Gesetz zur Umsetzung des Rahmenbeschlusses zum Europäischen Haftbefehl zu schaffen, bei dem auch dieser Aspekt berücksichtigt werden sollte. $\mathrm{Zu}$ beachten ist dann auch das Rückwirkungsverbot des Art. 103 II GG. Stichtag sollte das Datum des Inkrafttretens des neuen Umsetzungsgesetzes sein. Im übrigen könnte auch über die Zweiteilung zwischen dem Zulässigkeits- und dem Bewilligungsverfahren nachgedacht werden. 